\title{
Frauen übernehmen das Ruder
}

\begin{abstract}
Immer mehr Frauen entscheiden sich für den Arztberuf. Wie eine aktuelle Untersuchung des Zentralinstituts der Kassenärztlichen Versorgung (Zi) zeigt, stieg die Zahl der Vertragsärztinnen zwischen 2005 und 2014 von 37 Prozent auf 43,2 Prozent. In Krankenhäusern lag der Anteil der Frauen sogar bei 46 Prozent. Das sind zehn Prozentpunkte mehr als noch vor einem Jahrzehnt. Die Autoren der Studie gehen davon aus, dass sich der Trend zur Feminisierung in der Medizin künftig fortsetzen wird. Denn auch unter den Medizinstudierenden stieg der Frauenanteil in den vergangenen Jahren. Während 2004 noch etwa 53,6 Prozent aller Absolventen weiblich waren, lag der Anteil 2013 bereits bei 62,6 Prozent. Der Zi-Untersuchung zufolge behandeln Frauen anders als ihre männlichen Kollegen. Tendenziell würden sich Ärztinnen mehr Zeit für die Behandlung nehmen, hätten aber insgesamt kürzere Arbeitszeiten.
\end{abstract}

Der Blick in die Zahnmedizin zeigt deutliche Parallelen zur Ärzteschaft. Ob in der Zahnarztpraxis oder an der Hochschule, in allen Bereichen verzeichnen die Frauen ein Plus. Nach Angaben der Bundeszahnärztekammer (BZÄK) stieg der Anteil der Zahnärztinnen an allen zahnärztlich tätigen Personen zwischen 2000 und 2014 um 7,2 Prozentpunkte auf 43,5 Prozent. Für die nächsten Jahre prognostiziert das Institut für Zahnärzte (IDZ) bei der Anzahl behandelnd tätiger Zahnärzte sogar eine Umkehr in den Geschlechterverhältnissen. Das IDZ geht davon aus, dass von den im Jahr 2030 voraussichtlich insgesamt 70.400 behandelt tätigen Zahnärzten etwa 60 Prozent weiblich sein werden. Im Jahr 2014 lag der Anteil noch bei 46 Prozent. Auch die Zahlen an den Universitäten decken sich mit dem Trend aus der Ärzteschaft. Laut BZÄK-Statistik stieg der Anteil der Frauen unter den Zahnmedizinabsolventen seit dem Jahrtausendwechsel um etwa 16 Prozentpunkte auf 65,2 Prozent an.

Hier steht eine Anzeige.

Springer

\section{Medizinische Behandlung von Flüchtlingen \\ Flächendeckende Einführung der Gesundheitskarte ist ungewiss}

Die flächendeckende Einführung der Gesundheitskarte für Flüchtlinge droht zu scheitern. Zu diesem Ergebnis kommt eine aktuelle Studie der Bertelsmann Stiftung. „Nachdem der Bund die Finanzierungsverantwortung abgelehnt hat, müssen in den meisten Ländern weiterhin die Kommunen die Kosten der Gesundheitsversorgung tragen. Das hat zur Folge, dass die Einführung der Gesundheitskarte für Flüchtlinge insgesamt allenfalls schleppend vorankommt", heißt es im Fazit der Studie. Mit dem im September 2015 verabschiedeten Asylpaket I hatte die Bundesregierung die Einführung einer Gesundheitskarte für Flüchtlinge mit eingeschränktem Leistungsanspruch ermöglicht. Allerdings bleibt es den Bundesländern überlassen, ob sie die Karte einführen.

Die Studienautoren begründen den „Flickenteppich in der Gesundheitsversorgung von Asylsuchenden“ durch ungeklärte Finanzierungsfragen zwischen Bund und Ländern. Bisher haben lediglich sechs von sechzehn Bundesländern die Karte eingeführt oder einen verbindlichen Termin zur Einführung fest- gelegt. In den meisten Ländern tragen die Kommunen die Kosten der Gesundheitsversorgung der Asylsuchenden in den ersten 15 Monaten beziehungsweise bis zur Anerkennung des Asylgesuches. Zudem müssen kommunale Mitarbeiter darüber entschieden, ob eine Behandlung zulässig ist. In Ländern mit Gesundheitskarte sind die gesetzlichen Kassen für die Prüfung und Abrechnung zuständig. Die Behandlungskosten tragen auch dort weiterhin die Kommunen.

Laut der Studie befürchten die Kommunen mit der Einführung der Gesundheitskarte noch höhere Kosten. Beispiele dafür seien Nordrhein-Westfalen, wo lediglich 20 Kommunen die Gesundheitskarte einführen wollen, oder das Saarland, in dem alle Landkreise die Einführung verweigern. Mit Blick auf das Studienergebnis fordert die Kommunalexpertin der Bertelsmann Stiftung, Kirsten Witte: „Die medizinische Versorgung von Asylsuchenden und Flüchtlingen muss bundeseinheitlich geregelt werden." 